\title{
On the Existence of Configurations of Subspaces in a Hilbert Space with Fixed Angles
}

\author{
Natasha D. POPOVA and Yurii S. SAMOǏLENKO
}

Institute of Mathematics, National Academy of Sciences of Ukraine, 3 Tereshchenkivs'ka Str., Kyiv-4, 01601 Ukraine

E-mail: popova_n@yahoo.com,yurii_sam@imath.kiev.ua

Received December 01, 2005, in final form April 30, 2006; Published online May 29, 2006

Original article is available at http://www.emis.de/journals/SIGMA/2006/Paper055/

\begin{abstract}
For a class of $*$-algebras, where $*$-algebra $A_{\Gamma, \tau}$ is generated by projections associated with vertices of graph $\Gamma$ and depends on a parameter $\tau(0<\tau \leq 1)$, we study the sets $\Sigma_{\Gamma}$ of values of $\tau$ such that the algebras $A_{\Gamma, \tau}$ have nontrivial *-representations, by using the theory of spectra of graphs. In other words, we study such values of $\tau$ that the corresponding configurations of subspaces in a Hilbert space exist.
\end{abstract}

Key words: representations of $*$-algebras; Temperley-Lieb algebras

2000 Mathematics Subject Classification: 16G99; 20C08

\section{Introduction}

A number of papers is devoted to the study of $n$-tuples of subspaces in a Hilbert space. The interest to this problem arose in many respects due to its applications to problems of mathematical physics (see, e.g., [2] and the bibliography therein).

Unitary description of $n$-tuples of subspaces is $*$-wild problem when $n \geq 3$ (see [4]).

In the present paper we study the configurations of subspaces $H_{i}(i=1, \ldots, n)$ associated with the vertices of graph $\Gamma$, where an angle between any two of subspaces is fixed (see Section 3 ). It is convenient to consider such configurations of subspaces as $*$-representations of algebras generated by projections with relations of Temperley-Lieb type (see [3, 6, 9]). For tree $\Gamma$ the set $\Sigma_{\Gamma}$ (of those values of an "angle" $\tau$ where the corresponding configurations exist) is described in Section 4. This result is obtained by using the theory of graph spectra (needed notions and results are given in Section 2). For graphs containing cycles the situation is more complicated (see Remark 2).

\section{Necessary facts from theory of spectra of graphs}

We give some facts necessary for the exposition below, which can be found in [1]. Let $\Gamma$ be a finite undirected graph without multiple edges and loops. The adjacency matrix of a graph $\Gamma$, with vertex set $\{1, \ldots, n\}$, is $n \times n$ matrix $A_{\Gamma}=\left\|a_{i, j}\right\|_{i, j=1}^{n}$ with $a_{i, j}=1$ if there is an edge between $i$ and $j$, and $a_{i, j}=0$; otherwise $a_{i, i}=0 \forall i$. The eigenvalues of $A_{\Gamma}$ and the spectrum of $A_{\Gamma}$ are also called the eigenvalues and the spectrum of a graph $\Gamma$, respectively. The eigenvalues of $\Gamma$ are denoted by $\lambda_{1}, \ldots, \lambda_{n}$; they are real because $A_{\Gamma}$ is symmetric. We assume that $r=$ $\lambda_{1} \geq \lambda_{2} \geq \cdots \geq \lambda_{n}=q$. The largest eigenvalue $r=\lambda_{1}$ is called the index of a graph $\Gamma$.

Proposition 1. 1. If a graph $\Gamma$ contains at least one edge then $1 \leq r \leq n-1,-r \leq q \leq-1$, and $r=-q$ if and only if a component of $\Gamma$ with greatest index is a bipartite graph.

2. If $\Gamma$ is a connected graph then $2 \cos \frac{\pi}{n+1} \leq r$. 


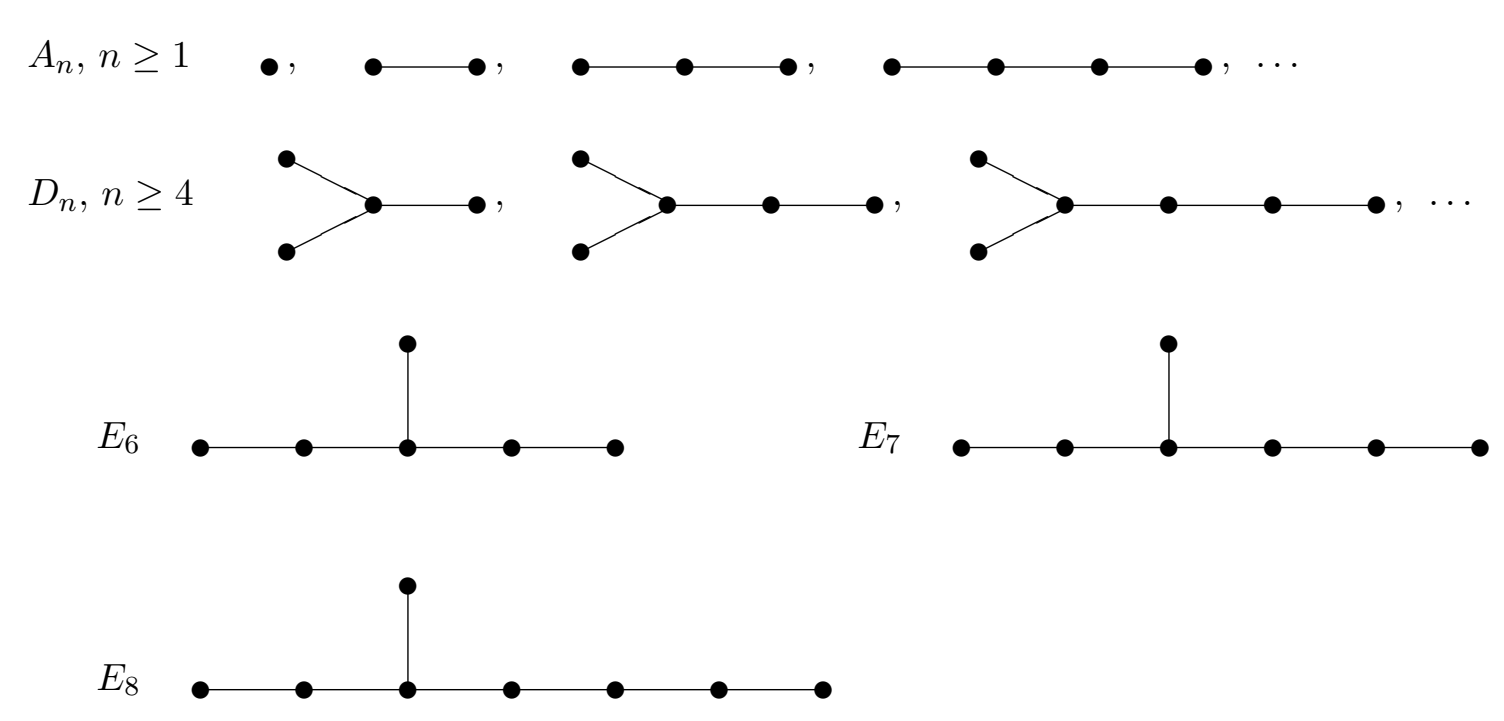

Figure 1. Dynkin diagrams $A_{n}, D_{n}, E_{6}, E_{7}, E_{8}$.

Remark 1. If $\Gamma$ is a tree then $r=-q$, as the tree is a bipartite graph.

We also need the following statement.

Theorem 1 (J.H. Smith). Let $\Gamma$ be a graph with index $r$. Then $r \leq 2(r<2)$ if and only if each component of $\Gamma$ is a subgraph (proper subgraph) of one of the graphs depicted in Fig. 2 which all have an index equal to 2.

Corollary 1 (For trees). Let $\Gamma$ be a tree with index $r$. Then

1. $r<2$ if and only if $\Gamma$ is one of the following graphs: $A_{n}, D_{n}, E_{6}, E_{7}, E_{8}$ (see Fig. 1).

2. $r=2$ if and only if $\Gamma$ is one of the following graphs: $\widetilde{D}_{n}, \widetilde{E}_{6}, \widetilde{E}_{7}, \widetilde{E}_{8}$ (see Fig. 2).

\section{Configurations of subspaces in a Hilbert space with fixed angles between them}

Let $H$ be a complex Hilbert space and let $H_{i}, H_{j} \subset H$ be its closed subspaces. We say that an angle between $H_{i}$ and $H_{j}$ is fixed and equals to $\varphi_{i, j} \in\left[0 ; \frac{\pi}{2}\right]$ if for the orthogonal projections $P_{H_{i}}, P_{H_{j}}$ on these subspaces we have

$$
P_{H_{i}} P_{H_{j}} P_{H_{i}}=\cos ^{2}\left(\varphi_{i, j}\right) P_{H_{i}} \quad \text { and } \quad P_{H_{j}} P_{H_{i}} P_{H_{j}}=\cos ^{2}\left(\varphi_{i, j}\right) P_{H_{j}}
$$

Having a finite undirected graph $\Gamma$ without multiple edges and loops with the numbers on its edges, we define the conditions on the configuration of subspaces in a Hilbert space as follows. The subspaces correspond to the vertices of a graph and an angle between any two of them is given by the number $\tau_{i, j}$ standing on the respective edge. If vertices are not adjacent we assume that the corresponding subspaces are orthogonal.

We consider the following questions:

1. For which values of the parameters $\tau_{i, j}$ the configuration associated with a graph $\Gamma$ exists.

2. Give the description of all irreducible configurations (associated with a fixed graph $\Gamma$ and an arrangement of numbers on its edges) up to a unitary transformation. 


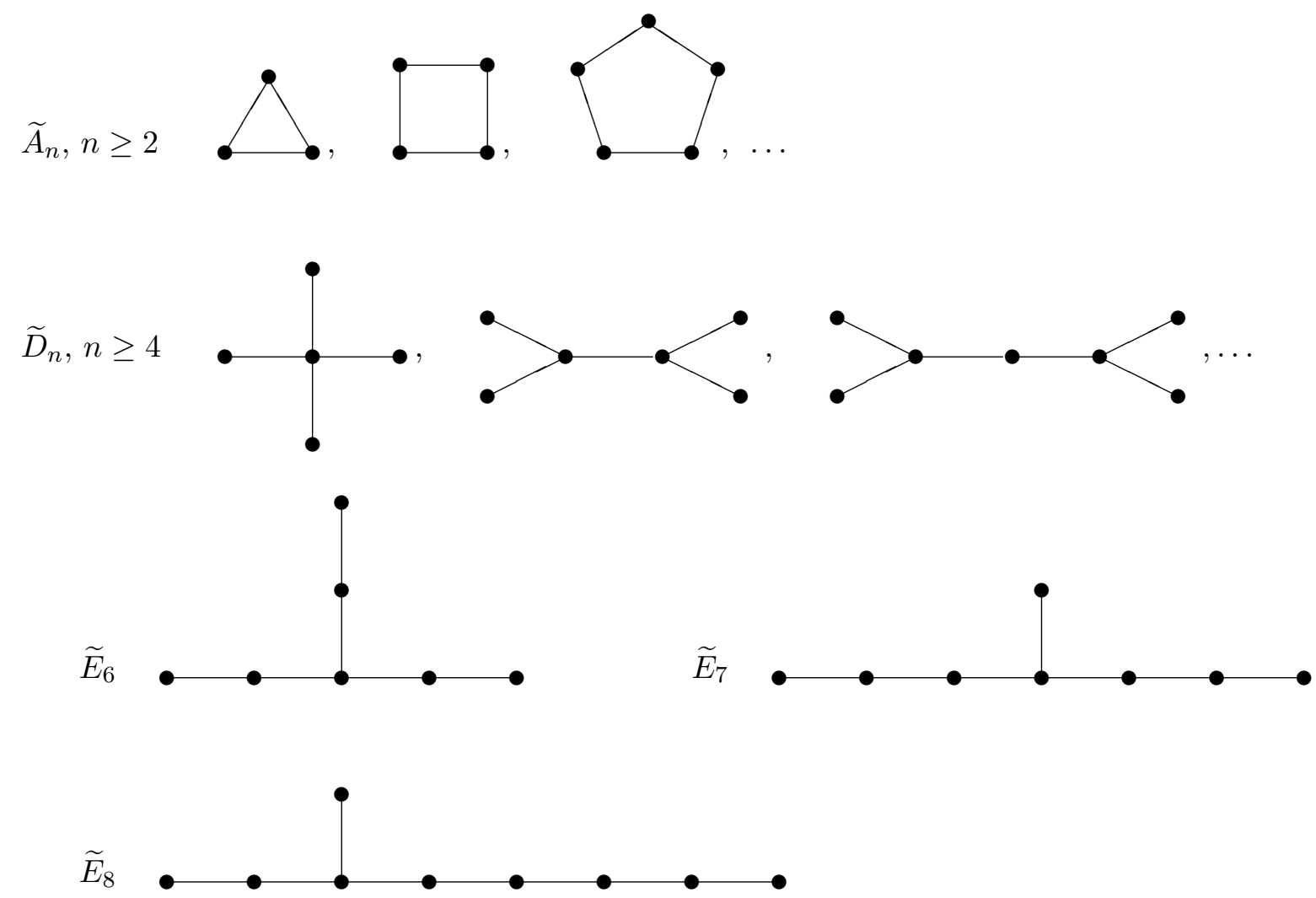

Figure 2. Extended Dynkin diagrams $\widetilde{A}_{n}, \widetilde{D}_{n}, \widetilde{E}_{6}, \widetilde{E}_{7}, \widetilde{E}_{8}$.

It should be noted that the subspaces corresponding to vertices from different components of $\Gamma$ are orthogonal, so we will consider only connected graphs.

These problems can be reformulated in terms of finding $*$-representations of $*$-algebras associated with $\Gamma$ with the numbers $\tau_{i, j}$ on the edges. Let $\Gamma$ be a finite, undirected, connected graph without multiple edges and loops, with $\Gamma_{0}\left(\left|\Gamma_{0}\right|=n\right)$ and $\Gamma_{1}$ the sets of the vertices and the edges respectively. Let $\tau: \Gamma_{1} \rightarrow(0,1)$ be the arrangement of numbers on its edges. We enumerate the vertices of $\Gamma$ by numbers $1, \ldots, n$ in any way and denote $\tau(i, j)=: \tau_{i, j}=\tau_{j, i}$.

Definition 1. $A_{\Gamma, \tau}$ is an $*$-algebra with 1 over $\mathbb{C}$ generated by projections $p_{1}, \ldots, p_{n}\left(p_{i}^{2}=p_{i}^{*}=\right.$ $\left.p_{i}, \forall i\right)$ with relations

$$
p_{i} p_{j} p_{i}=\tau_{i, j} p_{i} \quad \text { and } \quad p_{j} p_{i} p_{j}=\tau_{i, j} p_{j} \quad \text { if } \quad(i, j) \in \Gamma_{1}, \quad \text { and } \quad p_{i} p_{j}=p_{j} p_{i}=0 \text { otherwise. }
$$

Results on dimension of the algebra $A_{\Gamma, \tau}$ (in dependence on a graph $\Gamma$ ) can be found in $[7,8]$.

Below we suppose that $\Gamma$ is a tree. Then the $*$-algebra $A_{\Gamma, \tau}$ is finite dimensional and, therefore, does not have infinite dimensional irreducible $*$-representations.

\section{On the set of values of the parameters where $A_{\Gamma, \tau}$ has *-representations}

Let $\Gamma$ be a tree and $\mathcal{A}(\Gamma, \tau)=\left\|\mathcal{A}_{i, j}\right\|_{i, j=1}^{n}$ be the symmetric matrix with $\mathcal{A}_{i, i}=1 \forall i ; \mathcal{A}_{i, j}=\sqrt{\tau_{i, j}}$ if $(i, j) \in \Gamma_{1}$, and $\mathcal{A}_{i, j}=0$ otherwise.

Proposition 2. Let $\Gamma$ be a tree. Nontrivial $*$-representations of an algebra $A_{\Gamma, \tau}$ exist if and only if the matrix $\mathcal{A}(\Gamma, \tau)$ is positive semidefinite. Irreducible nontrivial *-representation is unique up to the unitary equivalence and its dimension is equal to the $\operatorname{rank}(\mathcal{A}(\Gamma, \tau))$. 
The proof one can find in [8].

In the following we suppose that $\tau$ is constant $\left(\tau_{i, j}=\tau \forall(i, j) \in \Gamma_{1}\right)$. The set of values of the parameter $\tau$ where $A_{\Gamma, \tau}$ has nontrivial $*$-representations we will denote by $\Sigma_{\Gamma}$.

Theorem 2. Let $\Gamma$ be a tree with index $r$. Then

$$
\Sigma_{\Gamma}=\left(0 ; \frac{1}{r^{2}}\right] \text {. }
$$

Proof. Indeed, $\mathcal{A}(\Gamma, \tau)=I+\sqrt{\tau} B_{\Gamma}$ where $I$ is $n \times n$ unit matrix and $B_{\Gamma}$ is the adjacency matrix of the tree $\Gamma$. The matrix $\mathcal{A}(\Gamma, \tau)$ is positive semidefinite if and only if its minimal eigenvalue is nonnegative, i.e., $1+\sqrt{\tau} q \geq 0$ (in the notations of Section 2) which is equivalent to $\tau \leq \frac{1}{q^{2}}$. For trees we know that $q=-r$ (see Remark 1), so the theorem is proved.

Example 1. Let us find the sets $\Sigma_{\Gamma}$ where graphs $\Gamma$ are Dynkin diagrams.

$$
\begin{aligned}
& \Sigma_{A_{n}}=\left(0 ; \frac{1}{4 \cos ^{2} \frac{\pi}{n+1}}\right], \quad \Sigma_{D_{n}}=\left(0 ; \frac{1}{4 \cos ^{2} \frac{\pi}{2(n-1)}}\right], \\
& \Sigma_{E_{6}}=\left(0 ; \frac{1}{4 \cos ^{2} \frac{\pi}{12}}\right], \quad \Sigma_{E_{7}}=\left(0 ; \frac{1}{4 \cos ^{2} \frac{\pi}{18}}\right], \quad \Sigma_{E_{8}}=\left(0 ; \frac{1}{4 \cos ^{2} \frac{\pi}{30}}\right] .
\end{aligned}
$$

Values of index of Dynkin diagrams can be found in [1].

Now we give some properties of $\Sigma_{\Gamma}$ ( $\Gamma$ is a tree) that immediately follow from Proposition 1 and Theorem 2 .

Proposition 3. Let $\Gamma$ be a tree with $n$ vertices. Then

$$
\text { 1) }\left(0 ; \frac{1}{(n-1)^{2}}\right] \subseteq \Sigma_{\Gamma}, \quad \text { 2) } \Sigma_{\Gamma} \subseteq\left(0 ; \frac{1}{4 \cos ^{2} \frac{\pi}{n+1}}\right]
$$

Remark 2. For graph $\Gamma$ that is not a tree situation is more complicated. For example, if the graph is a cycle with $n$ vertices, i.e. $\Gamma=\widetilde{A}_{n-1}$, we know that $\Sigma_{\Gamma}=\Sigma_{A_{n-1}}=\left(0 ; \frac{1}{4 \cos ^{2} \frac{\pi}{n}}\right]$ (see [5]). But the index of $\widetilde{A}_{n-1}$ is $r=2$ (see Theorem 1) and formula (1) does not hold. Moreover, it is known that all eigenvalues of $\widetilde{A}_{n-1}$ are of the form: $\lambda_{j}=2 \cos \frac{2 \pi}{n} j, j=1, \ldots, n$ (see [1]). Therefore, if $\mathrm{n}$ is even then no one eigenvalue $\lambda_{j}$ of the graph $\widetilde{A}_{n-1}$ makes the formula $\Sigma_{\Gamma}=\left(0 ; \frac{1}{\lambda_{j}^{2}}\right]$ true.

Next proposition follows directly from Corollary 1 and Theorem 2.

Proposition 4. Let $\Gamma$ be a tree. Then

1. $\max \Sigma_{\Gamma}>1 / 4$ if and only if $\Gamma$ is one of the following graphs: $A_{n}, D_{n}, E_{6}, E_{7}, E_{8}$.

2. $\max \Sigma_{\Gamma}=1 / 4$ if and only if $\Gamma$ is one of the following graphs: $\widetilde{D}_{n}, \widetilde{E}_{6}, \widetilde{E}_{7}, \widetilde{E}_{8}$.

3. For all other trees which are neither Dynkin diagrams nor extended Dynkin diagrams we have $\max \Sigma_{\Gamma}<1 / 4$. 
[1] Cvetković D.M., Doob M., Sachs H., Spectra of graphs. Theory and applications, Berlin, VEB Deutscher Verlag der Wissenschaften, 1980.

[2] Evans D.E., Kawahigashi Y., Quantum symmetries on operator algebras, Oxford University Press, 1998.

[3] Fan C.K., Green R.M., On the affine Temperley-Lieb algebras, J. London Math. Soc. (2), 1999, V.60, N 2, 366-380.

[4] Ostrovskyi V.L., Samoǐlenko Yu.S., Introduction to the theory of representations of finitely presented *-algebras. I. Representations by bounded operators, Harwood Acad. Publ., 1999.

[5] Popova N., On the algebra of Temperley-Lieb type, in Proceedings of Fourth International Conference "Symmetry in Nonlinear Mathematical Physics" (July 9-15, 2001, Kyiv), Editors A.G. Nikitin, V.M. Boyko and R.O. Popovych, Proceedings of Institute of Mathematics, Kyiv, 2002, V.43, Part 2, 486-489.

[6] Temperley H.N.V., Lieb E.H., Relations between "percolations" and "colouring" problems and other graph theoretical problems associated with regular planar lattices: some exact results for the percolation problem, Proc. Roy. Soc. London Ser. A, 1971, V.322, N 1549, 251-280.

[7] Vlasenko M., On the growth of an algebra generated by a system of projections with fixed angles, Methods Funct. Anal. Topology, 2004, V.10, N 1, 98-104.

[8] Vlasenko M., Popova N., On configurations of subspaces of Hilbert space with fixed angles between them, Ukrain. Mat. Zh., 2004, V.56, N 5, 606-615 (English transl.: Ukrainian Math. J., 2004, V.56, N 5, 730-740).

[9] Wenzl H., On sequences of projections, C. R. Math. Rep. Acad. Sci. Canada, 1987, V.9, N 1, 5-9. 